\title{
Vertex covers by edge disjoint cliques
}

\author{
Tom Bohman Alan Frieze* $\quad$ Miklós Ruszinkót \\ Lubos Thoma $a^{\ddagger}$ \\ Department of Mathematical Sciences, \\ Carnegie Mellon University.
}

June 6,2000

\begin{abstract}
Let $H$ be a simple graph having no isolated vertices. An $(H, k)$-vertex-cover of a simple graph $G=(V, E)$ is a collection $H_{1}, \ldots, H_{r}$ of subgraphs of $G$ satisfying

1. $H_{i} \cong H, \quad$ for all $i=1, \ldots, r$,

2. $\cup_{i=1}^{r} V\left(H_{i}\right)=V$,

3. $E\left(H_{i}\right) \cap E\left(H_{j}\right)=\emptyset, \quad$ for all $i \neq j$, and

4. each $v \in V$ is in at most $k$ of the $H_{i}$.

We consider the existence of such vertex covers when $H$ is a complete graph, $K_{t}, t \geq 3$, in the context of extremal and random graphs.
\end{abstract}

\section{Introduction}

Let $H$ be a simple graph having no isolated vertices. For the purposes of this discussion we say that the simple graph $G=(V, E)$ has property $\mathcal{C}_{H, k}$ if there is a collection $H_{1}, \ldots, H_{r}$ of subgraphs of $G$ satisfying

P1. $H_{i} \cong H, \quad$ for all $i=1, \ldots, r$,

P2. $\cup_{i=1}^{r} V\left(H_{i}\right)=V$,

P3. $E\left(H_{i}\right) \cap E\left(H_{j}\right)=\emptyset, \quad$ for all $i \neq j$, and

P4. each $v \in V$ is in at most $k$ of the $H_{i}$.

\footnotetext{
* Supported in part by NSF grant CCR-9530974.

$\dagger$ Permanent Address: Computer and Automation Research Institute of the Hungarian Academy of Sciences, Budapest, P.O.Box 63, Hungary-1518.

Research was partially supported by OTKA Grants T 030059 and T 29074, FKFP 0607/1999 and by the Bolyai Foundation.

${ }^{\ddagger}$ Supported in part by NSF grant DMS-9970622.
} 
We call the family $\left\{H_{1}, \ldots, H_{r}\right\}$ an $(H, k)$-vertex-cover of $G$. Thus when $k=1$ we ask for the existence of a partition of $V$ into vertex disjoint copies of $H$ i.e. the existence of an $H$-factor. In this case we assume the necessary divisibility condition, i.e. that $|V(H)|$ divides $|V|$. We study this property when $G$ is a random graph and also when $G$ is extremal w.r.t. minimum degree. In the main we will focus on the case where $H$ is a complete graph $K_{t}$ and denote our property by $\mathcal{C}_{t, k}$.

Random Graphs. The precise threshold for the occurrence of $\mathcal{C}_{2,1}$ i.e. the existence of a perfect matching was found by Erdős and Rényi [7] as part of a series of papers which laid the foundations of the theory of random graphs. The precise threshold for the occurrence of $\mathcal{C}_{3,1}$ i.e the existence of a vertex partition into triangles remains as one of the most challenging problems in this area (see, for example, the Appendix by Erdős to the monograph by Alon and Spencer [1]).

The thresholds for $H$-factors have been studied for example by Ruciński [15] and by Alon and Yuster [3]. For a graph $H$, let

$$
m_{1}(H)=\max \left(\frac{\left|E\left(H^{\prime}\right)\right|}{\left|V\left(H^{\prime}\right)\right|-1}\right)
$$

where the maximum is taken over all subgraphs $H^{\prime}$ of the graph $H$ with at least two vertices. In [15], Ruciński showed that the probability $p(n)=O\left(n^{-1 / m_{1}(H)}\right)$ is a sharp threshold for the property $\mathcal{C}_{H, 1}$ for any graph $H$ such that $m_{1}(H)>\delta(H)$ where $\delta(H)$ stands, as usual, for the minimum degree of the graph $H$. Note that, for example, $H$ being a complete graph is excluded. Hence, the first interesting open case is $H=K_{3}$. In [11], Krivelevich showed that the probability $p(n)=O\left(n^{-3 / 5}\right)$ is enough for the random graph to have a $K_{3}$-factor $\mathbf{w h p}^{1}$ and, in general, if $p(n)=O\left(n^{-2 t /(t-1)(t+2)}\right)$ then the random graph $G_{n, p}$ contains a $K_{t}$-factor whp (provided $t$ divides $n$ ).

An obvious necessary condition for the existence of a $\left(K_{t}, k\right)$-vertex-cover is that every vertex be incident with at least one copy of $K_{t}$.

Theorem 1. Let $m=\left(\begin{array}{c}n \\ 2\end{array}\right)\left((t-1) !\left(\log n+c_{n}\right)\right)^{1 /\left(\begin{array}{l}t \\ 2\end{array}\right)} n^{-2 / t}$. Then

$$
\lim _{n \rightarrow \infty} \operatorname{Pr}\left(G_{n, m} \text { contains a }\left(K_{t}, 2\right) \text {-vertex-cover }\right)= \begin{cases}0 & c_{n} \rightarrow-\infty \\ e^{-e^{-c}} & c_{n} \rightarrow c \\ 1 & c_{n} \rightarrow \infty\end{cases}
$$

(Here, $G_{n, m}$ stands for the probability space over the set of all graphs on $n$ vertices and with $m$ edges endowed with the uniform probability measure.) We will prove this as a consequence of the slightly stronger hitting time version. We consider the graph process $G_{m}=\left([n], E_{m}\right), m=0,1, \ldots,\left(\begin{array}{c}n \\ 2\end{array}\right)$, where $E_{0}=\emptyset$ and $G_{m}$ is obtained from $G_{m-1}$ by choosing $e_{m}$ randomly from $\left(\begin{array}{c}{[n]} \\ 2\end{array}\right) \backslash E_{m-1}$ and putting

\footnotetext{
${ }^{1}$ A sequence of events $\mathcal{E}_{n}$ occurs with high probability, whp, if $\operatorname{Pr}\left(\mathcal{E}_{n}\right)=1-o(1)$.
} 
$E_{m}=E_{m-1} \cup\left\{e_{m}\right\}$. We define two hitting times:

$\tau_{1}=\tau_{1}(t)=\min \left\{m:\right.$ Every $v \in[n]$ is contained in a copy of $K_{t}$ in $\left.G_{m}\right\}$,

$\tau_{2}=\tau_{2}(t)=\min \left\{m: G_{m}\right.$ contains a $\left(K_{t}, 2\right)$-vertex-cover $\}$.

Theorem 2. For every fixed $t \geq 3$,

$$
\lim _{n \rightarrow \infty} \operatorname{Pr}\left(\tau_{1}=\tau_{2}\right)=1 \text {. }
$$

Moreover, there exists whp a $\left(K_{t}, 2\right)$-vertex-cover of $G_{\tau_{2}}$ containing $(1+o(1)) \frac{n}{t}$ copies of $K_{t}$.

Remark 1. In fact, our proof of Theorem 2 implies that $G_{\tau_{2}}$ possesses whp a $\left(K_{t}, 2\right)$-vertex-cover containing at most $\left(\frac{1}{t}+\frac{1}{(\log n)^{1 / t}}\right) n$ copies of $K_{t}$.

Remark 2. Theorem 2 lends weight to the common conjecture that the threshold for a $K_{t}$-factor is $m$ of Theorem 1 .

We prove Theorem 2 in Section 2 and show how Theorem 1 follows from Theorem 2 in Section 3.

Extremal Graphs. For a graph $G$ on $n$ vertices what is the smallest minimum degree that insures $G$ has $\mathcal{C}_{t, k}$ ? For $t \geq 3$ and $k \geq 2$ let

$$
f(n, t, k)=\max \left\{d: \exists G \text { such that } \delta(G)=d,|V(G)|=n \text { and } G \notin \mathcal{C}_{t, k}\right\} .
$$

We will assume that $n$ is large with respect to $t$, but $k$ can be arbitrarily large. The smallest minimum degree that guarantees a $K_{t}$-factor (this would be, up to divisibility considerations, $f(n, t, 1)+1$ ) was established in the following deep theorem of Hajnal and Szemerédi [9].

Theorem 3 (Hajnal, Szemerédi). If $|V(G)|=n$ and $\delta(G) \geq\left(1-\frac{1}{t}\right) n$ then $G$ contains $\lfloor n / t\rfloor$ vertex-disjoint copies of $K_{t}$.

Our central result in this section is the following:

Theorem 4. Let $t \geq 3, k \geq 2, n \geq 6 t^{2}-4 t$ and

$$
n=q[(t-1) k+1]+r \text { where } 1 \leq r \leq(t-1) k+1 \text {. }
$$

Then

$$
n-q k-\left\lceil\frac{r}{t-1}\right\rceil \leq f(n, t, k) \leq n-q k--\left\lceil\frac{r}{t-1}\right\rceil+1
$$

Note that it follows from Theorem 4 that

$$
f(n, t, k)=\left\lfloor\frac{[(t-2) k+1] n}{(t-1) k+1}\right\rfloor+c
$$

where $c \in\{0,1,2\}$. It is tempting to believe that $f(n, t, k)$ equals the lower bound given in Theorem 4 . This is not the case in general. 
Theorem 5. Let $n \geq 6$ and $k \geq(n-1) / 2$.

$$
f(n, 3, k)=\left\lceil\frac{n}{2}\right\rceil \text {. }
$$

Note that the value of $f(n, 3, k)$ given in Theorem 5 equals the lower bound in Theorem 4 for $n$ even, but equals the upper bound for $n$ odd. (Here $q=0$ and $r=n)$.

For $H$ a simple graph with no isolated vertices and $G$ an arbitrary graph an $(H, \infty)$-vertex-cover of $G$ is a collection $H_{1}, \ldots, H_{r}$ of subgraphs of $G$ satisfying P1, P2 and P3. Thus, $G$ has an $(H, \infty)$-vertex-cover if and only if there exists a $k$ such that $G$ has a $(H, k)$-vertex-cover. To motivate our results on $(H, \infty)$ vertex-covers, we recall the following well-known extension of Theorem 3. Given an arbitrary graph $H$, Komlós, Sárközy and Szemerédi [13] showed that there is a constant $c$ (depending only on the graph $H$ ) such that if $\delta(G) \geq\left(1-\frac{1}{\chi(H)}\right) n$ for a graph $G$ on $n$ vertices, then there is a union of vertex-disjoint copies of $H$ covering all but at most $c$ vertices of $G$. Weakening the condition on $\delta(G)$ we show in the following theorem the existence of $(H, \infty)$-vertex-covers for graphs $H$ having the property that there is a vertex $u$ of $H$ such that $\chi(H \backslash\{u\})=\chi(H)-1 \geq 3$.

Theorem 6. Let $H$ be a graph such that $\chi(H) \geq 4$ and such that there is a vertex $u$ of $H$ with the property that $\chi(H \backslash\{u\})=\chi(H)-1$. Then for every $\epsilon>0$ and every graph $G$ on $n$ vertices, if $\delta(G) \geq\left(1-\frac{1}{\chi(H)-1}+\epsilon\right) n$, then $G$ has an $(H, \infty)$-vertex-cover provided $n$ is large enough.

Theorems 4, 5 and 6 are proved in Section 4.

\section{Proof of Theorem 2}

In this section we will use the following Chernoff bounds on the tails of the binomial random variable $B(n, p)$. For $0 \leq \epsilon \leq 1$ and $\theta>0$

$$
\begin{aligned}
\operatorname{Pr}(B(n, p) \leq(1-\epsilon) n p) & \leq e^{-\epsilon^{2} n p / 2} \\
\operatorname{Pr}(B(n, p) \geq(1+\epsilon) n p) & \leq e^{-\epsilon^{2} n p / 3} \\
\operatorname{Pr}(B(n, p) \geq \theta n p) & \leq(e / \theta)^{\theta n p}
\end{aligned}
$$

All Lemmas introduced in this section will be proven in the subsections that follow.

Let $t \geq 3$ be fixed. We construct a $\left(K_{t}, 2\right)$-vertex-cover in $G_{m}$ by dividing our graph process into 3 phases and using edges from different phases for different purposes. Before describing the phases, we make some preliminary definitions and the observation that we may restrict our attention to $G_{m}$ where $m$ lies in a small interval. Let $\alpha, \beta>0$ be constants such that

$$
\beta^{\left(\begin{array}{l}
t \\
2
\end{array}\right)}>19 / 20 \text { and } \alpha+\beta<1
$$


and let

$$
\begin{gathered}
m_{a}=\alpha\left(\begin{array}{l}
n \\
2
\end{array}\right)((t-1) ! \log n)^{1 /\left(\begin{array}{l}
t \\
2
\end{array}\right)} n^{-2 / t}, \text { and } \\
m_{b}=\beta\left(\begin{array}{l}
n \\
2
\end{array}\right)((t-1) ! \log n)^{1 /\left(\begin{array}{l}
t \\
2
\end{array}\right)} n^{-2 / t} .
\end{gathered}
$$

Furthermore, for $i=0,1$ let

$$
m_{i}=\left(\begin{array}{l}
n \\
2
\end{array}\right)((t-1) !(\log n-(1-2 i) \log \log n))^{1 /\left(\begin{array}{c}
t \\
2
\end{array}\right)} n^{-2 / t} .
$$

Lemma 1.

$$
\operatorname{Pr}\left(\tau_{1} \notin\left[m_{0}, m_{1}\right]\right)=o(1)
$$

We will use the term 'a collection of $K_{t}$ 's' in the graph $G$, for a family $\mathcal{A} \subseteq\left(\begin{array}{c}V(G) \\ t\end{array}\right)$ such that $G[S]$ is complete for all $S \in \mathcal{A}$. For such a collection $\mathcal{A}$ we set

$$
V(\mathcal{A})=\bigcup_{S \in \mathcal{A}} S \quad \text { and } \quad E(\mathcal{A})=\bigcup_{S \in \mathcal{A}}\left(\begin{array}{l}
S \\
2
\end{array}\right)
$$

say $\mathcal{A}$ 'covers' a vertex $v$ if $v \in V(\mathcal{A})$, and say $\mathcal{A}$ 'covers' a set of vertices $T$ if $T \subseteq V(\mathcal{A})$.

We are now ready to describe the 3 phases. In the first phase we simply choose $m_{a}$ edges uniformly at random, producing the graph $G^{1}=\left([n], E^{1}\right)$. Thus,

$$
G^{1}=G_{n, m_{a}} .
$$

In the second phase we form the graph $G^{2}=\left([n], E^{2}\right)$ by choosing $m_{b}$ edges uniformly at random. This is done independently of phase 1 and without knowledge of which edges were placed in phase 1 . Thus,

$$
G^{2}=G_{n, m_{b}},
$$

and a particular edge may appear in both $G^{1}$ and $G^{2}$. Let $F=E^{1} \cup E^{2}$ and $m_{-1}=|F|$. The third phase is the graph process $H_{i}=\left([n], F_{i}\right), i=m_{-1}, \ldots, m_{1}$ where $F_{m_{-1}}=F$ and $F_{i+1}$ is the union of $F_{i}$ and the set containing a single edge chosen uniformly at random from $\left(\begin{array}{l}n \\ 2\end{array}\right) \backslash F_{i}$. In other words, in the third phase we start with the collection of edges generated in phases 1 and 2 and then add new edges one at time until $m_{1}$ edges have been placed. Note that for $m_{a}+m_{b} \leq i \leq m_{1}$ the graphs $G_{i}$ and $H_{i}$ are identically distributed.

We henceforth assume that

$$
m_{a}+m_{b} \leq m \leq m_{1}
$$

and that every vertex in $H_{m}=G_{m}$ lies in at least one copy of $K_{t}$. We will show that

$$
\text { whp } G_{m} \text { has a }\left(K_{t}, 2\right) \text {-vertex-cover. }
$$


Theorem 2 follows from (5) and Lemma 1.

How do we construct the $\left(K_{t}, 2\right)$-vertex-cover? We first use the phase one edges to greedily cover as many vertices as possible with vertex disjoint $K_{t}$ 's. Let $\Xi$ be an arbitrary maximal collection of vertex disjoint $K_{t}$ 's in $G^{1}, X \subseteq[n]$ be the set of vertices not covered by $\Xi$, and

$$
r=\left\lceil\frac{n}{(\log n)^{1 / t}}\right\rceil .
$$

We can easily randomise this choice of $K_{t}$ 's so that $X$ is a random $|X|$-subset of $[n]$. This will be used in the proof of Lemma 4 .

Lemma 2. Let $G=G_{n, m_{a}}$.

$$
\operatorname{Pr}\left(\exists R \subset[n] \text { such that }|R|=r \text { and } G[R] \text { contains no } K_{t} ' s\right)=o(1) \text {. }
$$

It follows from Lemma 2 that whp

$$
|X| \leq r
$$

In other words, after using only a small fraction of the edges in $G_{m}$, only $o(n)$ vertices remain to be covered. We will use the phase 2 edges (as well as a handful of the phase 1 and phase 3 edges) to form a vertex disjoint collection of $K_{t}$ 's that covers $X$ but does not use any edge in $E(\Xi)$.

Before describing the vertex disjoint collection of $K_{t}$ 's that covers $X$, we make further definitions and preliminary observations. Our first observation concerns the random graph process $G_{m_{1}}$ alone. Let $\nu_{3}=4, \nu_{4}=3$ and $\nu_{i}=2$ for $i=5,6, \ldots$ We define a cluster to be a collection $\mathcal{C}=\left\{S_{1}, \ldots, S_{l}\right\}$ of $K_{t}$ 's in $G_{m_{1}}$ such that $l \leq 2 \nu_{t}$

$$
\begin{aligned}
& \kappa_{i} \geq 1 \quad \text { for } \quad i=2, \ldots, l \\
& \kappa_{i}=t \quad \Rightarrow \quad \kappa_{i-1}=1 \quad \wedge\left|S_{i} \cap S_{i-1}\right| \geq 2 \\
& \text { and } \quad\left|\left\{i: \kappa_{i} \neq 1\right\}\right|=\nu_{t}
\end{aligned}
$$

where

$$
\kappa_{i}=\left|S_{i} \cap\left(\bigcup_{j=1}^{i-1} S_{j}\right)\right| \quad \text { for } \quad i=2, \ldots, l .
$$

Roughly speaking, a cluster is a small collection of $K_{t}$ 's that have many or large pairwise intersections.

\section{Lemma 3.}

$$
\operatorname{Pr}\left(G_{m_{1}} \text { contains a cluster }\right)=o(1)
$$


We now turn our attention to the graph $G^{2}$. For $v \in[n]$ let $\Upsilon_{v}$ be the collection of $K_{t}$ 's in $G^{2}$ that contain $v$; to be precise,

$$
\Upsilon_{v}=\left\{S \in\left(\begin{array}{c}
{[n]} \\
t
\end{array}\right): v \in S \text { and }\left(\begin{array}{l}
S \\
2
\end{array}\right) \subseteq E^{2}\right\}
$$

Since $\Upsilon_{v}$ depends only on the graph $G^{2}$ while $X$ is small and depends only on the graph $G^{1}$, it is usually the case that no $V\left(\Upsilon_{v}\right)$ contains many members of $X$. To make this statement precise, we let

$$
q=\left\lceil\frac{\log n}{\log \log \log n}\right\rceil .
$$

\section{Lemma 4.}

$$
\operatorname{Pr}\left(\exists v \in[n] \text { such that }\left|V\left(\Upsilon_{v}\right) \cap X\right|>q\right)=o(1)
$$

We say that

$$
\begin{aligned}
& v \in[n] \text { is large if }\left|\Upsilon_{v}\right| \geq \frac{\log n}{20}, \text { and } \\
& v \in[n] \text { is small if }\left|\Upsilon_{v}\right|<\frac{\log n}{20} .
\end{aligned}
$$

With high probability the small vertices are, with respect to connections via $K_{t}$ 's, far apart. To make this statement precise, we define a chain to be a pair $u, v$ of distinct small vertices and a collection $S_{1}, S_{2}, S_{3}, S_{4} \in\left(\begin{array}{c}{[n]} \\ t\end{array}\right)$ of (not necessarily distinct) sets such that $u \in S_{1}, v \in S_{4}$,

$$
S_{1} \cap S_{2}, S_{2} \cap S_{3}, S_{3} \cap S_{4} \neq \emptyset, \quad \text { and } \quad\left(\begin{array}{c}
S_{i} \\
2
\end{array}\right) \subseteq E\left(G_{m_{1}}\right) \text { for } i=1,2,3,4 .
$$

\section{Lemma 5.}

$$
\operatorname{Pr}\left(G_{m_{1}} \text { contains a chain }\right)=o(1)
$$

We also note that no $K_{t}$ containing a small vertex intersects any other $K_{t}$ in more than a single vertex. A link is a small vertex $u \in[n]$ and distinct $S_{1}, S_{2} \in\left(\begin{array}{c}{[n]} \\ t\end{array}\right)$ such that $u \in S_{1},\left|S_{1} \cap S_{2}\right| \geq 2$, and $\left(\begin{array}{c}S_{1} \\ 2\end{array}\right),\left(\begin{array}{c}S_{2} \\ 2\end{array}\right) \subseteq E\left(G_{m_{1}}\right)$.

\section{Lemma 6.}

$$
\operatorname{Pr}\left(G_{m_{1}} \text { contains a link }\right)=o(1)
$$

Finally, let

$$
\begin{gathered}
X_{1}=\{v \in X: v \text { is small }\} \\
X_{2}=\{v \in X: v \text { is large }\}, \text { and } \\
\Phi=\left\{S \in\left(\begin{array}{c}
{[n]} \\
t
\end{array}\right):\left(\begin{array}{c}
S \\
2
\end{array}\right) \subseteq E\left(G_{m_{1}}\right) \text { and } S \cap X_{1} \neq \emptyset\right\} .
\end{gathered}
$$


We are now prepared to describe the remainder of the $\left(K_{t}, 2\right)$-cover.

We henceforth assume (6),

$$
\begin{gathered}
G_{m_{1}} \text { does not contain a cluster, } \\
\forall v \in[n] \quad\left|V\left(\Upsilon_{v}\right) \cap X\right| \leq q, \\
G_{m_{1}} \text { does not contain a chain, } \\
G_{m_{1}} \text { does not contain a link, }
\end{gathered}
$$

and that $n$ is sufficiently large (in a sense that is made clear below). We will show that there exist collections $\Xi_{1}$ and $\Xi_{2}$ of vertex disjoint $K_{t}$ 's in $G_{m}$ such that $\Xi_{1} \cup \Xi_{2}$ covers $X_{1} \cup X_{2}$ and

$$
V\left(\Xi_{1}\right) \cap V\left(\Xi_{2}\right)=\emptyset \text { and } E(\Xi) \cap E\left(\Xi_{1} \cup \Xi_{2}\right)=\emptyset \text {. }
$$

If follows from Lemmas 1, 2, 3, 4, 5 and 6 that (11) implies Theorem 2.

We cover $X_{1}$ in a rather crude way. Let $\Xi_{1}$ be an arbitrary collection of $K_{t}$ 's in $G_{m}$ that covers $X_{1}$. Note that the collection $\Xi_{1}$ uses edges from all 3 phases and that we make use of the fact that every vertex is contained in some $K_{t}$ in $G_{m}$ when forming $\Xi_{1}$. By (9), $\Xi_{1}$ is vertex disjoint.

We cover

$$
X_{2}^{\prime}:=X_{2} \backslash V\left(\Xi_{1}\right)
$$

in a more sophisticated way: we apply the Lovász Local Lemma. We first 'trim' the $\Upsilon_{v}$ 's. For $v \in X_{2}^{\prime}$ let $\Upsilon_{v}^{\prime}$ be the collection of sets in $S \in \Upsilon_{v}$ such that

$$
\begin{gathered}
S \cap X=\{v\} \\
T \in\left(\begin{array}{c}
{[n]} \\
t
\end{array}\right) \wedge\left(\begin{array}{c}
T \\
2
\end{array}\right) \subseteq E\left(G_{m_{1}}\right) \Rightarrow|S \cap T| \leq 1, \quad \text { and } \\
S \cap V(\Phi) \subseteq\{v\} .
\end{gathered}
$$

In words, we get $\Upsilon_{v}^{\prime}$ from $\Upsilon_{v}$ by throwing away those sets in $\Upsilon_{v}$ that contain an element of $X$ other than $v$, intersect another $K_{t}$ in more than one vertex, or contain a vertex of a $K_{t}$ that contains a small vertex. By (8) there are at most $q$ sets in $\Upsilon_{v}$ that contain an element of $X$ other than $v$. We will show

$$
\text { there are } \leq\left(\begin{array}{c}
2 \nu_{t} t \\
t
\end{array}\right) \text { sets in } \Upsilon_{v} \text { that intersect another } K_{t} \text { in } \geq 2 \text { vertices. }
$$

By (9) at most 1 set in $\Upsilon_{v}$ intersects $V(\Phi)$. Therefore, we may choose $\Theta_{v} \subseteq \Upsilon_{v}^{\prime}$ such that

$$
\left|\Theta_{v}\right|=\left\lceil\frac{\log n}{21}\right\rceil \quad \text { for all } \quad v \in X_{2}^{\prime}
$$

Proof of (13) Let $\hat{\Upsilon}_{v}$ denote the collection of $K_{t}$ 's in $\Upsilon_{v}$ which intersect another $K_{t}$ in more than one vertex. Let $B=V\left(\hat{\Upsilon}_{v}\right)$. We construct copies $X_{1}, X_{2}, \ldots, X_{l}$ 
of $K_{t}$ in $G_{m_{1}}$ as follows: Suppose we have constructed $X_{1}, X_{2}, \ldots, X_{k}$. Either (i) $B \subseteq V_{k}=V\left(X_{1} \cup X_{2} \cup \cdots \cup X_{k}\right)$ or (ii) $B \nsubseteq V_{k}$. In case (ii) choose $X_{k+1} \in \Upsilon_{v}$ which is not contained in $V_{k}$. If $\left|X_{k+1} \cap V_{k}\right|=1$ then choose $X_{k+2}$ where $\left|X_{k+2} \cap X_{k+1}\right| \geq 2$. If this process continues for $\nu_{t}$ iterations we will have produced a cluster. Thus $l \leq 2 \nu_{t}$ and $|B| \leq 2 t \nu_{t}$, which implies (13).

Now, consider the probability space in which each $v \in X_{2}^{\prime}$ chooses $S_{v} \in \Theta_{v}$ uniformly at random and independently of the other vertices. For $u \neq v \in X_{2}^{\prime}, S \in \Theta_{u}$ and $T \in \Theta_{v}$ such that $S \cap T \neq \emptyset$ let $A_{u, v, S, T}$ be the event that $S_{u}=S$ and $S_{v}=T$. These are the 'bad' events in our application of the Lovász Local Lemma. Clearly,

$$
\operatorname{Pr}\left(A_{u, v, S, T}\right)=\frac{1}{\left|\Theta_{v}\right|\left|\Theta_{u}\right|} \leq\left(\frac{21}{\log n}\right)^{2}=: p .
$$

Events $A_{u_{1}, u_{2}, S_{1}, S_{2}}$ and $A_{v_{1}, v_{2}, T_{1}, T_{2}}$ are dependent if and only if

$$
\left\{u_{1}, u_{2}\right\} \cap\left\{v_{1}, v_{2}\right\} \neq \emptyset .
$$

Thus, the degree in the dependency graph is bounded above by

$$
\begin{aligned}
d: & =2 \max _{u \in X_{2}^{\prime}} \sum_{S \in \Theta_{u}} \sum_{v \in X_{2}^{\prime}}\left|\left\{T \in \Theta_{v}: S \cap T \neq \emptyset\right\}\right| \\
& \leq 2 \max _{u \in X_{2}^{\prime}} \sum_{w \in V\left(\Theta_{u}\right)}\left|\Upsilon_{w} \cap X\right| \\
& \leq 2 t q\left\lceil\frac{\log n}{21}\right\rceil \quad \text { by }(8) \\
& \leq \frac{t(\log n)^{2}}{10 \log \log \log n} .
\end{aligned}
$$

It follows from (15) and (16) that

$$
p d \leq \frac{45}{\log \log \log n}=o(1) .
$$

Thus, for $n$ sufficiently large, it follows from the Lovász Local Lemma that there exists a vertex disjoint collection $\Xi_{2}$ of $K_{t}$ 's in $G^{2}$ that covers $X_{2}^{\prime}$ but covers no vertex in $V\left(\Xi_{1}\right)$.

It remains to show that

$$
E(\Xi) \cap E\left(\Xi_{1} \cup \Xi_{2}\right)=\emptyset
$$

This is an immediate consequence of (10) and (12). We have established (11) and completed the proof.

\section{$2.1 \quad$ Proof of Lemma 1}

Let $p_{i}=m_{i} /\left(\begin{array}{l}n \\ 2\end{array}\right)$ for $i=0,1$. 
We first apply Janson's inequality to show that whp every vertex in $G_{n, p_{1}}$ is contained in a copy of $K_{t}$ (we follow the notation of [1, pages 95 and 96]). Let $v$ be a fixed vertex and let and $Z$ denote the number of copies of $K_{t}$ in $G$ which are incident with $v$. Next let $S_{1}, S_{2}, \ldots, S_{\left(\begin{array}{c}n-1 \\ t-1\end{array}\right)}$ be an enumeration of the copies of $K_{t}$ in $K_{n}$ which contain $v$. Letting $B_{j}$ be the event $\left(\begin{array}{c}S_{j} \\ 2\end{array}\right) \subseteq E\left(G_{n, p_{i}}\right)$, we have

$$
\mu=\sum_{j=1}^{\left(\begin{array}{c}
n-1 \\
t-1
\end{array}\right)} \operatorname{Pr}\left(B_{j}\right)=\left(\begin{array}{c}
n-1 \\
t-1
\end{array}\right) p_{1}^{\left(\begin{array}{c}
t \\
2
\end{array}\right)}=(\log n+\log \log n)(1+O(1 / n))
$$

and

$$
\begin{aligned}
\Delta & =\sum_{\left|S_{j} \cap S_{k}\right| \geq 2} \operatorname{Pr}\left(B_{j} \cap B_{k}\right) \\
& =\left(\begin{array}{l}
n-1 \\
t-1
\end{array}\right) \sum_{r=2}^{t-1}\left(\begin{array}{l}
t-1 \\
r-1
\end{array}\right)\left(\begin{array}{l}
n-t \\
t-r
\end{array}\right) p_{i}^{2\left(\begin{array}{c}
t \\
2
\end{array}\right)-\left(\begin{array}{c}
r \\
2
\end{array}\right)} \\
& =O\left(\sum_{r=2}^{t-1} n^{2 t-r-1-\frac{2}{t}\left(2\left(\begin{array}{l}
t \\
2
\end{array}\right)-\left(\begin{array}{l}
r \\
2
\end{array}\right)\right)+o(1)}\right) \\
& =O\left(n^{2 t^{-1}-1+o(1)}\right) .
\end{aligned}
$$

Then, by Janson's inequality, we have

$$
\begin{aligned}
\operatorname{Pr}(Z=0) & \leq \exp \left\{-\mu+\frac{1}{1-\epsilon} \frac{\Delta}{2}\right\} \\
& =\frac{1}{n \log n} \exp \left\{O\left(n^{-1+o(1)}\right)+O\left(n^{2 t^{-1}-1+o(1)}\right)\right\} \\
& =o(1 / n) .
\end{aligned}
$$

It follows that

$$
\operatorname{Pr}\left(\exists u \in[n]: u \text { is not contained in a copy of } K_{t} \text { in } G_{n, p_{1}}\right)=o(1) .
$$

The event $\left\{\exists u \in[n]: u\right.$ is not contained in a copy of $\left.K_{t}\right\}$ is monotone decreasing and so (20) implies that whp every vertex in $[n]$ is contained in a copy of $K_{t}$ in $G_{n, m_{1}}$. In other words, $\tau_{1} \leq m_{1}$ whp.

We now turn to the random graph $G_{n, p_{0}}$ in order to establish our almost sure lower bound on $\tau_{1}$. For $v \in[n]$ let $Z_{v}$ be the number of $K_{t}$ 's in $G_{n, p_{0}}$ that contain $v$, and let $Y$ denote the number of vertices $v$ such that $Z_{v}=0$. Since

$$
M=\left(1-p_{0}\right)^{\left(\begin{array}{c}
n-1 \\
t-1
\end{array}\right)}=(1+o(1)) \frac{\log n}{n}
$$

is a lower bound on $\operatorname{Pr}\left(Z_{v}=0\right)$ for each $v \in[n]$, we have

$$
\mathbf{E}(Y) \geq(1+o(1)) \log n .
$$


We now show that $\operatorname{Var}(Y)$ is small. Indeed,

$$
\operatorname{Pr}\left(Z_{1}=Z_{2}=0\right) \leq \operatorname{Pr}\left(\mathcal{E}_{1}\right)+\operatorname{Pr}\left(\overline{\mathcal{E}}_{2} \overline{\mathcal{E}}_{3} \mid \overline{\mathcal{E}}_{1}\right)
$$

where, if $N_{i}$ is the set of neighbors of $i$ in $G_{n, p_{0}}$,

$$
\begin{gathered}
\mathcal{E}_{1}=\left\{\left(\left(1-n^{-\frac{1}{4 t}}\right) n p_{0} \leq\left|N_{1}\right|,\left|N_{2}\right| \leq 2 n p_{0}\right)\right. \\
\left.\left.\vee\left(\left|N_{1} \cap N_{2}\right| \geq n^{-\frac{1}{4 t}} n p_{0}\right\}\right)\right\} \\
\mathcal{E}_{2}=\left\{G_{n, p_{0}} \text { contains a copy } H \text { of } K_{t-1} \text { such that } H \subseteq N_{1}\right\} \\
\mathcal{E}_{3}=\left\{G_{n, p_{0}} \text { contains a copy } H \text { of } K_{t-1} \text { such that } H \subseteq N_{2} \backslash N_{1}\right\} .
\end{gathered}
$$

Applying (2)-(4) we get,

$$
\operatorname{Pr}\left(\mathcal{E}_{1}\right) \leq 5 \exp \left\{-n^{1-\frac{5}{2 t}+o(1)}\right\}
$$

Note that

$$
\operatorname{Pr}\left(\overline{\mathcal{E}_{2}} \wedge \overline{\mathcal{E}_{3}} \mid N_{1}, N_{2}\right)=\operatorname{Pr}\left(\overline{\mathcal{E}_{2}} \mid N_{1}, N_{2}\right) \operatorname{Pr}\left(\overline{\mathcal{E}_{3}} \mid N_{1}, N_{2}\right)
$$

because, conditioning on $N_{1}$ and $N_{2}$, these events depend on disjoint sets of edges. Let $W_{1}$ and $W_{2}$ be fixed sets that satisfy

$$
\left(1-\frac{1}{n^{\frac{1}{4 t}}}\right) n p_{0} \leq\left|W_{1}\right| \leq 2 n p_{0} \text { and }\left(1-\frac{2}{n^{\frac{1}{4 t}}}\right) \leq\left|W_{2} \backslash W_{1}\right| \leq 2 n p_{0} .
$$

It follows from another application of Janson's inequality that

$$
\begin{aligned}
\operatorname{Pr}\left(\overline{\mathcal{E}_{2}} \mid N_{1}=W_{1},\right. & \left.N_{2} \backslash N_{1}=W_{2}\right), \operatorname{Pr}\left(\overline{\mathcal{E}_{3}} \mid N_{1}=W_{1}, N_{2} \backslash N_{1}=W_{2}\right) \\
\leq & \exp \left\{-\log n+\log \log n+O\left(n^{-\frac{1}{4 t}+o(1)}\right)+O\left(n^{-1+\frac{2}{t}+o(1)}\right)\right\} .
\end{aligned}
$$

Therefore,

$$
\operatorname{Pr}\left(Z_{1}=Z_{2}=0\right)=\frac{\log ^{2} n}{n^{2}}(1+o(1)),
$$

and it follows from (21) that

$$
\operatorname{Var}(Y)=o\left(\log ^{2} n\right)
$$

It then follows from Chebyshev's inequality that

$$
\operatorname{Pr}(Y=0)=o(1)
$$

Since the event $\{Y=0\}$ is monotone increasing, it follows from (24) that

$\operatorname{Pr}\left(\right.$ every vertex in $G_{n, m_{0}}$ is contained in a copy of $\left.K_{t}\right)=o(1)$.

In other words, we have shown that whp $\tau_{1}>m_{0}$. 


\subsection{Proof of Lemma 2}

Let $p_{a}=m_{a} /\left(\begin{array}{c}n \\ 2\end{array}\right)$ and consider the random graph $G=G_{n, p_{a}}$. For $S \in\left(\begin{array}{c}{[n]} \\ t\end{array}\right)$ let $B_{S}$ be the event that the induced graph $G[S]$ is complete. For $R$ a fixed subset of $[n]$ such that

$$
|R|=r=\left\lceil\frac{n}{(\log n)^{1 / t}}\right\rceil
$$

let the random variable $X_{R}$ be the number of copies of $K_{t}$ contained in $R$. We clearly have

$$
\begin{aligned}
\mu & :=\mathbf{E}\left[X_{R}\right] \\
& =\sum_{S \in\left(\begin{array}{l}
R \\
t
\end{array}\right)} \operatorname{Pr}\left(B_{S}\right) \\
& =\left(\begin{array}{l}
r \\
t
\end{array}\right) p_{a}^{\left(\begin{array}{c}
t \\
2
\end{array}\right)} \\
& =\left(\begin{array}{l}
r \\
t
\end{array}\right) \frac{\alpha^{\left(\begin{array}{l}
t \\
2
\end{array}\right)}(t-1) ! \log n}{n^{t-1}} \\
& =\frac{r^{t}}{t !}(1+O(1 / r)) \frac{\alpha^{\left(\begin{array}{c}
t \\
2
\end{array}\right)}(t-1) !}{n^{t-1}} \log n \\
& =\Omega(n)
\end{aligned}
$$

We apply Janson's inequality (again, we follow the notation of [1]) to show that $\operatorname{Pr}\left(X_{R}=0\right)$ is small. In order to do so, we must bound the parameter $\Delta$.

$$
\begin{aligned}
\Delta & =\sum_{S, T \in\left(\begin{array}{c}
R \\
t
\end{array}\right): 2 \leq|S \cap T| \leq t-1} \operatorname{Pr}\left(B_{S} \wedge B_{T}\right) \\
& =\left(\begin{array}{l}
r \\
t
\end{array}\right) \sum_{i=2}^{t-1}\left(\begin{array}{c}
t \\
i
\end{array}\right)\left(\begin{array}{c}
r-t \\
t-i
\end{array}\right) p_{a}^{2\left(\begin{array}{c}
t \\
2
\end{array}\right)-\left(\begin{array}{c}
i \\
2
\end{array}\right)} \\
& =\sum_{i=2}^{t-1} O\left(n^{2 t-i-\frac{2}{t}\left(2\left(\begin{array}{c}
t \\
2
\end{array}\right)-\left(\begin{array}{c}
i \\
2
\end{array}\right)\right)+o(1)}\right) \\
& =\sum_{i=2}^{t-1} O\left(n^{2+\frac{i(i-1)}{t}-i+o(1)}\right) \\
& =O\left(n^{2 / t+o(1)}\right) .
\end{aligned}
$$

Thus, Janson's inequality gives

$$
\operatorname{Pr}\left(X_{R}=0\right) \leq e^{-c_{1} n}
$$


where $c_{1}$ is a positive constant. Applying the first moment method, we have

$$
\begin{aligned}
\operatorname{Pr}\left(\bigvee_{R \in\left(\begin{array}{c}
{[n]} \\
r
\end{array}\right)}\left\{X_{R}=0\right\}\right) & \leq\left(\begin{array}{c}
n \\
r
\end{array}\right) e^{-c_{1} n} \\
& \leq\left(\frac{n e}{r}\right)^{r} e^{-c_{1} n} \\
& =\exp \left\{r\left(1+\frac{\log \log n}{t}\right)-c_{1} n\right\} \\
& =o(1)
\end{aligned}
$$

Since this event is monotone, the same holds for $G_{n, m_{a}}$.

\subsection{Proof of Lemma 3}

Let $\mathcal{C}=\left\{S_{1}, \ldots, S_{l}\right\}$ be a fixed collection of $K_{t}$ 's in $K_{n}$ such that $l \leq 2 \nu_{t}$

$$
\begin{aligned}
& \kappa_{i} \geq 1 \quad \text { for } \quad i=2, \ldots, l \\
& \kappa_{i}=t \quad \Rightarrow \quad \kappa_{i-1}=1 \wedge\left|S_{i} \cap S_{i-1}\right| \geq 2 \\
& \text { and } \quad\left|\left\{i: \kappa_{i} \neq 1\right\}\right|=\nu_{t}
\end{aligned}
$$

where

$$
\kappa_{i}=\left|S_{i} \cap\left(\bigcup_{j=1}^{i-1} S_{j}\right)\right| \quad \text { for } \quad i=2, \ldots, l .
$$

Let $a=|V(\mathcal{C})|$ and $b=|E(\mathcal{C})|$.

\section{Claim 7.}

$$
a-\frac{2 b}{t}<-\frac{1}{t}
$$

Proof. We observe this difference as we 'build' the collection $\mathcal{C}$ one $K_{t}$ at a time. For $j=1, \ldots, l$ let $\mathcal{C}_{j}=\left\{S_{1}, \ldots, S_{j}\right\}, a_{j}=\left|V\left(\mathcal{C}_{j}\right)\right|, b_{j}=\left|E\left(\mathcal{C}_{j}\right)\right|$ and $d_{j}=$ $a_{j}-2 b_{j} / t$. Note that

$$
d_{1}=1
$$

and

$$
d_{i+1}-d_{i} \leq\left(t-\kappa_{i+1}\right)-\frac{2}{t}\left(\left(\begin{array}{l}
t \\
2
\end{array}\right)-\left(\begin{array}{c}
\kappa_{i+1} \\
2
\end{array}\right)\right)=\left(\kappa_{i+1}-1\right)\left(\frac{\kappa_{i+1}}{t}-1\right) .
$$

Thus

$$
\begin{gathered}
\kappa_{i+1}=1 \quad \Rightarrow \quad d_{i+1}-d_{i}=0 \\
\text { and } \quad 2 \leq \kappa_{i+1} \leq t-1 \quad \Rightarrow \quad d_{i+1}-d_{i} \leq \frac{2}{t}-1 .
\end{gathered}
$$


Furthermore, it follows from (25) that

$$
\kappa_{i+1}=t \quad \Rightarrow \quad b_{i+1} \geq b_{i}+t-2 \quad \Rightarrow \quad d_{i+1}-d_{i} \leq-\frac{2(t-2)}{t} .
$$

Since (by (28) and (29)) the difference $a_{i}-2 b_{i} / t$ decreases by at least $1-2 / t$ whenever $\kappa_{i+1} \neq 1$, it follows from (26) that $a-2 b / t=d_{l}<-1 / t$.

Let $\mathcal{E}_{i}$ be the event that there exists a cluster in $G_{m_{1}}$ with a vertex set of cardinality $i$, and let $b_{i}$ be the minimum number of edges in a cluster on $i$ vertices. With $p_{m_{1}}=m_{1} /\left(\begin{array}{l}n \\ 2\end{array}\right)$ we have

$$
\begin{aligned}
\operatorname{Pr}\left(\mathcal{E}_{i}\right) & \leq\left(\begin{array}{c}
n \\
i
\end{array}\right) 2^{\left(\begin{array}{c}
i \\
t^{\prime}
\end{array}\right) p_{m_{1}}^{b_{i}}} \\
& =O\left(n^{i-\frac{2 b_{i}}{t}+o(1)}\right) \\
& =O\left(n^{-\frac{1}{t}+o(1)}\right) .
\end{aligned}
$$

The lemma then follows from the fact that the cardinality of the vertex set of a cluster is at most $2 \nu_{t} t$, a constant depending only on $t$.

\subsection{Proof of Lemma 4}

We first argue that whp

$$
\left|\Upsilon_{v}\right| \leq 4 \log n \quad \text { for all } v \in[n] .
$$

We can calculate in $G_{n, p_{b}}$ where $p_{b}=m_{b} / N, N=\left(\begin{array}{l}n \\ 2\end{array}\right)$ and then use monotonicity to translate the result to $G^{2}$. It follows from Lemma 3 and (13) that whp after removing $O(1) K_{t}$ 's from $\Upsilon_{v}$ we have a collection $\tilde{\Upsilon}_{v}$ of $K_{t}$ 's which are disjoint except for there containing $v$. So in $G_{n, p_{b}}$

$$
\operatorname{Pr}\left(\left|\tilde{\Upsilon}_{v}\right| \geq \kappa=3.9 \log n\right) \leq \frac{\left(\begin{array}{c}
n-1 \\
t-1
\end{array}\right)^{\kappa}}{\kappa !} p_{b}^{\kappa\left(\begin{array}{c}
t \\
2
\end{array}\right)} \leq \frac{(\log n)^{\kappa}}{\kappa !} \leq(e / 3.9)^{3.9 \log n}=o\left(n^{-3 / 2}\right) .
$$

This verifies (30).

Now fix a vertex $v$. Then $\left|V\left(\Upsilon_{v}\right)\right|<4 t \log n$ and $|X| \leq r$. Also, $X$ and $V\left(\Upsilon_{v}\right)$ are chosen independently. It follows that

$$
\begin{aligned}
\operatorname{Pr}\left(\left|V\left(\Upsilon_{v}\right) \cap X\right| \geq q\right) & \leq \frac{\left(\begin{array}{c}
4 t \log n \\
q
\end{array}\right)\left(\begin{array}{c}
n-q \\
r-q
\end{array}\right)}{\left(\begin{array}{l}
n \\
r
\end{array}\right)} \\
& \leq\left(\frac{4 t e r \log n}{q n}\right)^{q} \\
& \leq\left(\frac{4 t e \log \log \log n \log n}{(\log n)^{(t+1) / t}}\right)^{\log n / \log \log \log n} \\
& =O\left(n^{-A}\right)
\end{aligned}
$$


for any constant $A>0$.

There are $n$ choices for $v$ and the lemma follows.

\subsection{Proof of Lemmas 5 and 6}

Let

$$
p=((t-1) ! \log n)^{1 /\left(\begin{array}{l}
t \\
2
\end{array}\right)} n^{-2 / t} \quad \text { and } \quad p_{m_{1}}=\frac{m_{1}}{\left(\begin{array}{l}
n \\
2
\end{array}\right)} .
$$

The main work of this section is the following claim.

Claim 8. Let $H=(A, B)$ be a fixed graph whose vertex set $A$ is a subset of $[n]$, and let $x, y \in A$ be distinct fixed vertices. If $b:=|B|$ and $a:=|A| \leq 4$ then

1. $\operatorname{Pr}\left((x\right.$ is small $\left.) \wedge\left(H \subseteq G_{m_{1}}\right)\right)=O\left(p_{m_{1}}^{b} n^{-3 / 4}\right)$

2. $\operatorname{Pr}\left((x\right.$ and $y$ are small $\left.) \wedge\left(H \subseteq G_{m_{1}}\right)\right)=O\left(p_{m_{1}}^{b} n^{-3 / 2}\right)$

Proof. We only prove 2 ; the proof of 1 is both similar and easier. Let $\mathcal{R}_{x}$ be the event that $x$ is small, $\mathcal{R}_{y}$ be the event that $y$ is small, and let $\mathcal{R}_{H}$ be the event $B \subseteq E\left(G_{m_{1}}\right)$. Furthermore, let

$$
N_{x}=\left\{v \in[n]: x \sim_{G^{2}} v\right\} \backslash A \quad \text { and } \quad N_{y}=\left\{v \in[n]: y \sim_{G^{2}} v\right\} \backslash\left(A \cup N_{x}\right),
$$

$G_{x}$ be the induced graph $G^{2}\left[N_{x}\right]$, and $G_{y}=G^{2}\left[N_{y}\right]$. Finally, let $\epsilon>0$ be a constant such that

$$
\beta+\epsilon<1 \text { and }(\beta-\epsilon)^{\left(\begin{array}{l}
t \\
2
\end{array}\right)} \geq \frac{3}{4}+\frac{1}{20}(1+\log 20) .
$$

Case 1. $t=3$

We condition on the event that $N_{x}$ and $N_{y}$ are of nearly the expected size. Let $\mathcal{R}_{1}$ be the event that

$$
(\beta-\epsilon) n p \leq\left|N_{x}\right|,\left|N_{y}\right| \leq(\beta+\epsilon) n p,
$$

and $\mathcal{R}_{2}$ be the event that

$$
\left|E\left(G_{x}\right)\right|,\left|E\left(G_{y}\right)\right| \leq \frac{\log n}{20} .
$$

We have

$$
\operatorname{Pr}\left(\mathcal{R}_{H} \wedge \mathcal{R}_{x} \wedge \mathcal{R}_{y}\right) \leq \operatorname{Pr}\left(\mathcal{R}_{2} \mid \mathcal{R}_{1} \wedge \mathcal{R}_{H}\right) \operatorname{Pr}\left(\mathcal{R}_{H}\right)+\operatorname{Pr}\left(\overline{\mathcal{R}_{1}}\right)
$$

Now the Chernoff bounds show that in $G_{n, p_{m_{1}}}$ we have

$$
\operatorname{Pr}\left(\overline{\mathcal{R}}_{1}\right)=O\left(\exp \left\{-n^{1-\frac{2}{t}+o(1)}\right\}\right)
$$


and we can inflate this by $O(n)$ to show the same for $G_{m_{1}}$.

Then, where $N=\left(\begin{array}{l}n \\ 2\end{array}\right)$

$$
\begin{aligned}
& \left.\operatorname{Pr}\left(\mathcal{R}_{H}\right) \leq\left(\begin{array}{c}
a \\
2
\end{array}\right)\right)\left(\begin{array}{c}
N-b \\
m_{1}-b
\end{array}\right) /\left(\begin{array}{c}
N \\
m_{1}
\end{array}\right) \\
& =O\left(p_{m_{1}}^{b}\right) \text {. }
\end{aligned}
$$

To bound $\operatorname{Pr}\left(\mathcal{R}_{2}\right)$ we condition on $N_{x}=S, N_{y}=T$ satisfying (32), where $S, T$ are fixed subsets of $[n]$. Now let $\hat{\mathcal{R}}_{2}$ denote the event

$$
|E(S)|,|E(T)| \leq \frac{\log n}{20} .
$$

We show that for $\gamma \geq \beta-\epsilon$, in $G_{n, \gamma p}$ we have

$$
\operatorname{Pr}_{\gamma p}\left(\hat{\mathcal{R}}_{2}\right)=O\left(n^{-3 / 2}\right) .
$$

The monotonicity of $\hat{\mathcal{R}}_{2}$ plus the concentration of the number of edges of $G_{n, \gamma p}$ around $\gamma N p$ then allows us to assert (37) for $G^{2}$. Indeed, then

$$
O\left(n^{-3 / 2}\right)=\mathbf{P r}_{\gamma p}\left(\hat{\mathcal{R}}_{2}\right)=\sum_{m}\left(\begin{array}{l}
N \\
m
\end{array}\right)(\gamma p)^{m}(1-\gamma p)^{N-m} \mathbf{P r}_{m}\left(\hat{\mathcal{R}}_{2}\right)
$$

and so taking $\beta-\epsilon \leq \gamma$ we see that if $\operatorname{Pr}_{m_{1}}\left(\hat{\mathcal{R}}_{2}\right) \geq A n^{-3 / 2}$ then $\operatorname{Pr}_{\gamma p}\left(\hat{\mathcal{R}}_{2}\right) \geq$ $A n^{-3 / 2} / 2$.

The random variable $X=\left|E\left(G_{x}\right)\right|$ (in $G_{n, \gamma p}$ ) is a binomial random variable $B(s, p)$ where $s=\left(\begin{array}{c}|S| \\ 2\end{array}\right)$, having mean $\mu$ where

$$
(\beta-\epsilon)^{3} \log n<\mu<(\beta+\epsilon)^{3} \log n .
$$

So,

$$
\begin{aligned}
\operatorname{Pr}_{\gamma p}\left(X \leq \frac{\log n}{20}\right) & \leq \sum_{l=0}^{\left\lfloor\frac{\log n}{20}\right\rfloor}\left(\begin{array}{l}
s \\
l
\end{array}\right)(\gamma p)^{l}(1-\gamma p)^{s-l} \\
& \leq(1+o(1)) \sum_{l=0}^{\left\lfloor\frac{\log n}{20}\right\rfloor} e^{-\mu} \frac{\mu^{l}}{l !} \\
& \leq 2 e^{-\mu} \frac{\mu^{\left\lfloor\frac{\log n}{20}\right\rfloor}}{\left\lfloor\frac{\log n}{20}\right\rfloor !} \\
& \leq 3 \exp \left\{-\log n\left((\beta-\epsilon)^{3}-\frac{1}{20}(1+\log 20)\right)\right\} \\
& \leq 3 n^{-3 / 4}
\end{aligned}
$$

We apply the same argument to $\left|E\left(G_{y}\right)\right|$ (adding the appropriate conditioning on the number of edges within $N_{y}$ ). The proof now follows from (34) - (37). 
Case 2. $t \geq 4$

We bound $\operatorname{Pr}\left(\mathcal{R}_{x} \wedge \mathcal{R}_{y} \wedge \mathcal{R}_{H}\right)$ by conditioning on the event that the neighborhoods of $x$ and $y$ are of nearly the expected size and have nearly the expected number of edges. Let $\mathcal{R}_{3}$ is the event that

$$
\begin{gathered}
(\beta-\epsilon) p n \leq\left|N_{x}\right|,\left|N_{y}\right| \leq(\beta+\epsilon) p n \\
(\beta-\epsilon) p\left(\begin{array}{c}
\left|N_{x}\right| \\
2
\end{array}\right) \leq\left|E\left(G_{x}\right)\right| \leq(\beta+\epsilon) p\left(\begin{array}{c}
\left|N_{x}\right| \\
2
\end{array}\right), \text { and } \\
(\beta-\epsilon) p\left(\begin{array}{c}
\left|N_{y}\right| \\
2
\end{array}\right) \leq\left|E\left(G_{y}\right)\right| \leq(\beta+\epsilon) p\left(\begin{array}{c}
\left|N_{y}\right| \\
2
\end{array}\right)
\end{gathered}
$$

Let $\mathcal{R}_{4}$ be the event that both $G_{x}$ and $G_{y}$ contain fewer than $\frac{\log n}{20}$ copies of $K_{t}$. We now bound the probability of $\mathcal{R}_{x} \wedge \mathcal{R}_{y} \wedge \mathcal{R}_{H}$ as follows:

$$
\begin{aligned}
\operatorname{Pr}\left(\mathcal{R}_{x} \wedge \mathcal{R}_{y} \wedge \mathcal{R}_{H}\right) & \leq \operatorname{Pr}\left(\mathcal{R}_{4} \mid \mathcal{R}_{H} \wedge \mathcal{R}_{3}\right) \operatorname{Pr}\left(\mathcal{R}_{H}\right)+\operatorname{Pr}\left(\overline{\mathcal{R}_{3}}\right) \\
& \leq \operatorname{Pr}\left(\mathcal{R}_{4} \mid \mathcal{R}_{H} \wedge \mathcal{R}_{3}\right) O\left(p_{m_{1}}^{b}\right)+O\left(\exp \left\{-n^{1-\frac{2}{t}+o(1)}\right\}\right)
\end{aligned}
$$

We bound $\operatorname{Pr}\left(\mathcal{R}_{4} \mid \mathcal{R}_{H} \wedge \mathcal{R}_{3}\right)$ by an application of the Poisson approximation on the number of $K_{t}$ 's in the random graph $G_{n, m}$ given by Theorem 6.1 of [8, page 68]. We let $n^{\prime}$ and $m^{\prime}$ be integers satisfying

$$
\begin{gathered}
(\beta-\epsilon) p n \leq n^{\prime} \leq(\beta+\epsilon) p n, \text { and } \\
(\beta-\epsilon) p\left(\begin{array}{c}
n^{\prime} \\
2
\end{array}\right) \leq m^{\prime} \leq(\beta+\epsilon) p\left(\begin{array}{c}
n^{\prime} \\
2
\end{array}\right)
\end{gathered}
$$

and condition on the event that $\left|N_{x}\right|=n^{\prime}$ and $\left|E\left(G_{x}\right)\right|=m^{\prime}$. Note that under this conditioning $G_{x}$ can be viewed as the random graph $G_{n^{\prime}, m^{\prime}}$. Following the notation of [8], we have

$$
\frac{1}{2}\left(n^{\prime}\right)^{2-\frac{2}{t-2}} \omega_{1} \leq m^{\prime} \leq \frac{1}{2}\left(n^{\prime}\right)^{2-\frac{2}{t-2}} \omega_{2}
$$

where

$$
\omega_{1}=(\beta-\epsilon)^{\frac{t}{t-2}}((t-1) ! \log n)^{1 /\left(\begin{array}{c}
t-1 \\
2
\end{array}\right)}
$$

and

$$
\omega_{2}=(\beta+\epsilon)^{\frac{t}{t-2}}((t-1) ! \log n)^{1 /\left(\begin{array}{c}
t-1 \\
2
\end{array}\right)} .
$$

Let $X=X_{K_{t}}$ be the number of copies of $K_{t}$ in $G_{n^{\prime}, m^{\prime}}$. The expected number of such $K_{t}$ 's, $\lambda:=\mathbf{E}[X]$, is then bounded as follows:

$$
(\beta-\epsilon)^{\left(\begin{array}{l}
t \\
2
\end{array}\right)} \log n \leq \lambda \leq(\beta+\epsilon)^{\left(\begin{array}{l}
t \\
2
\end{array}\right)} \log n .
$$


It then follows from Theorem 6.1 of [8] that

$$
\begin{aligned}
\operatorname{Pr}\left(X \leq \frac{\log n}{20}\right) & \leq(1+o(1)) \sum_{k=0}^{\left\lfloor\frac{\log n}{20}\right\rfloor} e^{-\lambda} \frac{\lambda^{k}}{k !} \\
& \leq 2 e^{-\lambda} \frac{\lambda^{\left\lfloor\frac{\log n}{20}\right\rfloor}}{\left\lfloor\frac{\log n}{20}\right\rfloor !} \\
& \leq 2 e^{-\lambda}\left(\frac{20 e \lambda}{\log n}\right)^{\frac{\log n}{20}} \\
& \leq 2 \exp \left\{-(\beta-\epsilon)^{\left.\left(\begin{array}{c}
t \\
2
\end{array}\right) \log n\right\}(20 e)^{\frac{\log n}{20}}}\right. \\
& =2 \exp \left\{-\log n\left((\beta-\epsilon)^{\left(\begin{array}{c}
t \\
2
\end{array}\right)}-\frac{1}{20}(1+\log 20)\right)\right\} \\
& \leq 2 n^{-3 / 4}
\end{aligned}
$$

With (38) this completes the proof.

Proof of Lemma 5. Let $\mathcal{S}_{1}$ be the event that there is a chain in $G_{m_{1}}$. For a fixed collection $\mathcal{A}$ of $K_{t}$ 's in $K_{n}$ and distinct $u, v \in[n]$ which define a possible chain, it follows from an argument along the line of the proof of Claim 7 that

$$
|V(\mathcal{A})| \leq 1+\frac{2|E(\mathcal{A})|}{t}
$$

and it follows from Claim 8 that

$$
\operatorname{Pr}\left((u \text { and } v \text { are small }) \wedge E(\mathcal{A}) \subseteq E\left(G_{m_{1}}\right)\right) \leq O\left(p_{m_{1}}^{|E(\mathcal{A})|} n^{-3 / 2}\right) .
$$

Applying the first moment method we have

$$
\begin{aligned}
\operatorname{Pr}\left(\mathcal{S}_{1}\right) & \leq\left(\begin{array}{c}
n \\
2
\end{array}\right) \sum_{i=t}^{4 t-3}\left(\begin{array}{c}
n-2 \\
i-2
\end{array}\right) 2^{\left(\begin{array}{l}
i \\
t
\end{array}\right)} O\left(p_{m_{1}}^{\frac{(i-1) t}{2}} n^{-3 / 2}\right) \\
& \leq \sum_{i=t}^{4 t-3} O\left(n^{i-\frac{2}{t} \frac{(i-1) t}{2}-\frac{3}{2}+o(1)}\right) \\
& \leq \sum_{i=t}^{4 t-3} O\left(n^{-\frac{1}{2}+o(1)}\right) \\
& =o(1)
\end{aligned}
$$

Proof of Lemma 6 . Let $\mathcal{S}_{2}$ be the event that there is a link in $G_{m_{1}}$. For fixed $S, T \in\left(\begin{array}{c}{[n]} \\ t\end{array}\right)$ such that $|S \cap T| \geq 2$ and $x \in S \cup T$ it follows from Claim 8 that

$$
\operatorname{Pr}\left((x \text { is small }) \wedge\left(\begin{array}{l}
S \\
2
\end{array}\right) \cup\left(\begin{array}{l}
T \\
2
\end{array}\right) \subseteq E\left(G_{m_{1}}\right)\right)=O\left(p_{m_{1}}^{\left(\begin{array}{c}
t \\
2
\end{array}\right)-\left(\begin{array}{c}
|S \cap T| \\
2
\end{array}\right)} n^{-3 / 4}\right) .
$$


Applying the first moment method we have

$$
\begin{aligned}
\operatorname{Pr}\left(\mathcal{S}_{2}\right) & \leq n\left(\begin{array}{c}
n-1 \\
t-1
\end{array}\right) \sum_{i=2}^{t-1}\left(\begin{array}{l}
t \\
i
\end{array}\right)\left(\begin{array}{l}
n-t \\
t-i
\end{array}\right) O\left(\begin{array}{c}
2\left(\begin{array}{c}
t \\
2
\end{array}\right)-\left(\begin{array}{c}
i \\
2
\end{array}\right) \\
m_{1}
\end{array} n^{-3 / 4}\right) \\
& \leq \sum_{i=2}^{t-1} O\left(n^{2 t-i-2(t-1)+\frac{2}{t}\left(\begin{array}{c}
i \\
2
\end{array}\right)-\frac{3}{4}+o(1)}\right) \\
& \leq \sum_{i=2}^{t-1} O\left(n^{\frac{5}{4}-i+\frac{i(i-1)}{t}+o(1)}\right) \\
& =o(1)
\end{aligned}
$$

\section{Proof of Theorem 1.}

For a graph $G$ and a vertex $v$, we defined prior to $(21) Z_{v}(G)=Z_{v}$ to be the number of $K_{t}$ 's in $G$ that contain $v$ and $Y(G)=Y$ to be the number of vertices $u$ with $Z_{u}=0$.

In view of Theorem 2 we need only prove that

$$
\lim _{n \rightarrow \infty} \operatorname{Pr}\left(Y\left(G_{n, m}\right)=0\right)= \begin{cases}0 & c_{n} \rightarrow-\infty \\ e^{-e^{-c}} & c_{n} \rightarrow c \\ 1 & c_{n} \rightarrow \infty\end{cases}
$$

Using Theorem 2 of Luczak [14] we can derive (41) from the more easily obtained

$$
\lim _{n \rightarrow \infty} \operatorname{Pr}\left(Y\left(G_{n, p}\right)=0\right)= \begin{cases}0 & c_{n} \rightarrow-\infty \\ e^{-e^{-c}} & c_{n} \rightarrow c \\ 1 & c_{n} \rightarrow \infty\end{cases}
$$

where $p=m /\left(\begin{array}{l}n \\ 2\end{array}\right)$. Furthermore we need only consider the case $c_{n} \rightarrow c$ as the others follow by monotonicity. Equation $(42)$ can be proved by showing that $Y\left(G_{n, p}\right)$ is asymptotically Poisson. In particular we need only show that for $k=O(1)$,

$$
\lim _{n \rightarrow \infty} n^{k} \operatorname{Pr}\left(Z_{i}\left(G_{n, p}\right)=0,1 \leq i \leq k\right)=e^{-c k}
$$

and then apply e.g. Theorem 20 of Bollobás [5].

Equation (43) follows from

$$
\operatorname{Pr}\left(Z_{i}\left(G_{n, p}\right)=0 \mid Z_{j}\left(G_{n, p}\right)=0,1 \leq j<i\right) \sim \frac{e^{-c}}{n}
$$

for $1 \leq i \leq k$.

Using $N_{j}$ to denote the neighbourhood of $j$ in $G_{n, p}$ we let 
- $\nu_{1}$ denote the number of $K_{t-1}$ in $N_{i} \backslash \bigcup_{j=1}^{i-1} N_{j}$.

- $\nu_{2}$ denote the number of $K_{t-1}$ in $N_{i}$ which use a vertex of $\bigcup_{j=1}^{i-1} N_{j}$.

We then let $\mathcal{C}_{i}=\left\{Z_{j}\left(G_{n, p}\right)=0,1 \leq j<i\right\}$ and write

$$
\operatorname{Pr}\left(Z_{i}\left(G_{n, p}\right)=0 \mid \mathcal{C}_{i}\right)=\operatorname{Pr}\left(\nu_{1}=0 \mid \mathcal{C}_{i}\right)\left(1-\operatorname{Pr}\left(\nu_{2} \neq 0 \mid \nu_{1}=0, \mathcal{C}_{i}\right)\right) .
$$

Then $\operatorname{Pr}\left(\nu_{1}=0 \mid \mathcal{C}_{i}\right) \sim e^{-c} / n$ follows from Janson's inequality and $\operatorname{Pr}\left(\nu_{2} \neq 0 \mid\right.$ $\left.\nu_{1}=0, \mathcal{C}_{i}\right) \leq \operatorname{Pr}\left(\nu_{2} \neq 0\right)=o(1 / n)$ follows from the FKG inequality and a first moment calculation.

\section{Proofs of Theorems $4-6$}

We prove Theorem 4 via an application of the following theorem of Hajnal and Szemerédi. For $k \leq n$ the Turán graph $T_{k}(n)$ is the complete $k$-partite graph on $n$ vertices where the parts in the vertex partition have cardinalities

$$
\left\lfloor\frac{n}{k}\right\rfloor,\left\lfloor\frac{n+1}{k}\right\rfloor, \ldots,\left\lfloor\frac{n+k-1}{k}\right\rfloor .
$$

In other words, the parts in the partition are as near as possible to being equal (i.e. the partition is a so-called equipartition). Below we use the following theorem proved by Hajnal and Szemerédi (cf. Theorem 3).

Theorem 7 (Hajnal, Szemerédi). If $G$ is a graph on $n$ vertices having maximum degree $\Delta(G)=\Delta$ then

$$
G \subseteq T_{\Delta+1}(n)
$$

For a graph $G$, let $\bar{G}$ be the complement of $G$. It is easy to see that Theorem 7 is equivalent to

Theorem 8. If $G$ is a graph on $n$ vertices having minimum degree $\delta(G)=\delta$ then

$$
\overline{T_{n-\delta}(n)} \subseteq G
$$

Let a $\left(K_{t}, l\right)$-vertex-cover be a $K_{t}$-vertex-cover in which each vertex appears in at most $l$ copies of $K_{t}$.

Proof of Theorem 4. We establish the lower bound by example. Consider the complete $t$-partite graph on $n$ vertices having parts $V_{1}, \ldots, V_{t}$ such that $\left|V_{1}\right|=q$ and

$$
\left|V_{2}\right|, \ldots,\left|V_{t}\right| \in\left\{l q+\left\lceil\frac{r}{t-1}\right\rceil, l q+\left\lfloor\frac{r}{t-1}\right\rfloor\right\}
$$


If $q=0$ then $G$ contains no $t$-clique and therefore has no $\left(K_{t}, l\right)$-vertex-cover. If $q>0$ then, by the definition of $r$, there exists $V_{i}$ such that $\left|V_{i}\right|>q l$, and $G$ has no $\left(K_{t}, l\right)$-vertex-cover.

Suppose $G$ is a graph on $n$ vertices having

$$
\delta(G) \geq n-q l-\left\lceil\frac{r}{t-1}\right\rceil+2 .
$$

Let

$$
s=q l+\left\lceil\frac{r}{t-1}\right\rceil-2 .
$$

It follows from Theorem 8 that $\overline{T_{s}(n)} \subseteq G$. In words, there exists an equipartition $V(G)=V_{1} \cup \cdots \cup V_{s}$ such that the induced graph $G\left[V_{i}\right]$ is complete for $i=1, \ldots s$. We will show that the collection of cliques $G\left[V_{1}\right], \ldots, G\left[V_{s}\right]$ can be transformed into a $\left(K_{t}, l\right)$-vertex-cover.

\section{Claim 9.}

$$
t-1 \leq\left|V_{i}\right| \leq t \text { for } i=1, \ldots, s .
$$

Proof. We merely observe that $s(t-1)<n$ while $s t \geq n$.

$$
\begin{aligned}
{\left[q l+\left[\frac{r}{t-1}\right]-2\right](t-1) } & \leq q l(t-1)+\left(\frac{r}{t-1}+1\right)(t-1)-2(t-1) \\
& \leq q l(t-1)+r-(t-1) \\
& <n .
\end{aligned}
$$

On the other hand,

$$
\begin{aligned}
{\left[q l+\left\lceil\frac{r}{t-1}\right\rceil-2\right] t } & \geq\left[q l+\frac{r}{t-1}-2\right] t \\
& =n+q(l-1)+\frac{r}{t-1}-2 t .
\end{aligned}
$$

Now, since $n \geq 6 t^{2}-4 t$, at least one of the following holds:

- $r \geq 2 t(t-1)$

- $q \geq 2 t$

- $q(t-1) l \geq 4 t(t-1)$.

In any of these situations, the expression in (46) is greater than or equal to $n$.

If follows from Claim 9 that we may assume that for some $m$ we have $\left|V_{1}\right|=\cdots=$ $\left|V_{m}\right|=t-1$ and $\left|V_{m+1}\right|=\cdots=\left|V_{s}\right|=t$. 


\section{Claim 10.}

$$
m<(l-1)(q+1) .
$$

Proof. Since $V_{1}, \ldots, V_{s}$ is a partition, we must have $(t-1) m+t(s-m)=n$. However,

$$
\begin{aligned}
(t-1)(l-1)(q+1)+t & {\left[q l+\left[\frac{r}{t-1}\right]-2-(l-1)(q+1)\right] } \\
& =q[(t-1) l+1]+t\left[\frac{r}{t-1}\right]+1-l-2 t \\
& \leq q[(t-1) l+1]+t\left(\frac{r}{t-1}+\frac{t-2}{t-1}\right)+1-l-2 t \\
& \leq n+\frac{1}{t-1}+t \frac{t-2}{t-1}+1-2 t \\
& =n-t \\
& <n
\end{aligned}
$$

We transform $G\left[V_{1}\right], \ldots, G\left[V_{s}\right]$ into a $\left(K_{t}, l\right)$-vertex-cover by expanding the clique $V_{i}$ by one vertex for $i=1, \ldots, m$. To be precise, we will show that there exist $x_{1}, \ldots, x_{m} \in V(G)$ such that

1. $x_{i} \sim v \quad \forall v \in V_{i}$,

2. $\left|\left\{x_{i}: x_{i}=v\right\}\right| \leq l-1 \quad \forall v \in V(G)$,

3. $x_{i} \in V_{j} \Rightarrow x_{j} \notin V_{i}$,

4. $x_{i} \notin V_{i}$.

Note that the third condition must be included to prevent two of the expanded cliques from containing a common edge. For $i=1, \ldots, m$ let

$$
A_{i}=\left\{v \in V(G) \backslash V_{i}: v \sim u \quad \forall u \in V_{i}\right\}
$$

Claim 11. $\left|A_{i}\right| \geq q+t$ for $i=1, \ldots m$.

Proof. Since, for $v \in V_{i}$,

$$
\begin{aligned}
\left|\left\{x \in V(G) \backslash V_{i}: x \not \nu\right\}\right| & \leq n-1-\delta(G) \\
& \leq q l+\left\lceil\frac{r}{t-1}\right\rceil-3,
\end{aligned}
$$


we have

$$
\begin{aligned}
\mid\left\{x \in V(G) \backslash V_{i}: \exists v \in V_{i}\right. & \text { such that } x \not v\} \mid \\
& \leq(t-1)\left[q l+\left[\frac{r}{t-1}\right]-3\right] \\
& \leq q l(t-1)+(t-1)\left(\frac{r}{t-1}+\frac{t-2}{t-1}\right)-3(t-1) \\
& =q l(t-1)+r-2 t+1 .
\end{aligned}
$$

Therefore

$$
\begin{aligned}
\left|A_{i}\right| & =\left|V(G) \backslash V_{i}\right|-\mid\left\{x \in V(G) \backslash V_{i}: \exists v \in V_{i} \text { such that } x \not v\right\} \mid \\
& \geq n-(t-1)-[q l(t-1)+r-2 t+1] \\
& =q+t
\end{aligned}
$$

Now, we choose the $x_{i}$ 's one at a time in an order $x_{1}=x_{i_{1}}, x_{i_{2}}, \ldots x_{i_{m}}$ as follows. Suppose $x_{i_{1}}, \ldots, x_{i_{k}}$ have been chosen.

$$
\text { If } x_{i_{k}} \in V_{j} \text { and } j \notin\left\{i_{1}, \ldots, i_{k}\right\} \text { then } j=i_{k+1} \text {. }
$$

Otherwise $i_{k+1}$ is chosen arbitrarily from $\{j: 1 \leq j \leq m\} \backslash\left\{i_{1}, \ldots, i_{k}\right\}$. In other words, we chose the $x_{i}$ 's in an order such that at most one $x_{i}$ falls in $V_{j}$ before $x_{j}$ is chosen. For $k=1, \ldots, m$ let

$$
U_{k}=\left\{v \in V(G):\left|\left\{1 \leq j<k: x_{i_{j}}=v\right\}\right|=l-1\right\} .
$$

In words, $U_{k}$ is the set of vertices that satisfy 2 . with equality after $x_{i_{1}}, \ldots, x_{i_{k-1}}$ have been determined. Thus, we must have $x_{i_{k}} \notin U_{k}$. By Claim 10

$$
\left|U_{k}\right| \leq\left\lfloor\frac{m-1}{l-1}\right\rfloor<q+1 .
$$

For $k=1, \ldots, m$ let

$$
R_{k}=\bigcup_{1 \leq j<k: x_{i_{j}} \in V_{k}} V_{i_{j}} .
$$

(Note that the union here is over zero or one set only). By condition 3. we must have $x_{i_{k}} \notin R_{k}$. By the construction of the ordering given in (47),

$$
\left|R_{k}\right| \leq t-1 \text {. }
$$

An arbitrary $x_{i_{k}} \in\left(A_{i_{k}} \backslash U_{k}\right) \backslash R_{k}$ satisfies 1, 2, and 3. By (48), (49) and Claim 11 such an element exists. 
Proof of Theorem 6. Let $\epsilon>0$ and let $G$ be a graph on $n$ vertices with $\delta(G)=\delta \geq\left(1-\frac{1}{\chi(H)-1}+\epsilon\right) n$. We show that any collection of edge disjoint copies of $H$ that does not cover $V(G)$ can be extended to cover at least one new vertex. To be precise, we show that if a family $\mathcal{F}=\left\{\Gamma_{1}, \ldots, \Gamma_{m}\right\}$ of copies of $H$ in $G$ and a vertex $v \in V(G)$ satisfy

$$
\begin{aligned}
& m<n, \\
& \Gamma_{i}=\left(V\left(\Gamma_{i}\right), E\left(\Gamma_{i}\right)\right) \text { are copies of } H \text { in } G \text { for all } i, \\
& E\left(\Gamma_{i}\right) \cap E\left(\Gamma_{j}\right)=\emptyset \text { for all } i \neq j,
\end{aligned}
$$

and

$$
v \notin \cup_{i=1}^{m} V\left(\Gamma_{i}\right),
$$

then there exists a family $\mathcal{F}^{\prime}=\left\{\Upsilon_{1}, \ldots, \Upsilon_{l}\right\}$ such that for all $i \Upsilon_{i}=\left(V\left(\Upsilon_{i}\right), E\left(\Upsilon_{i}\right)\right)$ are copies of $H$ in $G$

$$
E\left(\Upsilon_{i}\right) \cap E\left(\Upsilon_{j}\right)=\emptyset \text { for all } i \neq j
$$

and

$$
\cup_{i=1}^{l} V\left(\Upsilon_{i}\right) \supseteq\left(\bigcup_{i=1}^{m} V\left(\Gamma_{i}\right)\right) \cup\{v\} .
$$

Note that we include the possibility of $m=0$. Clearly, an inductive argument based on (50) and (51) above implies the theorem. Further, we may assume $m<n$ in (50). Suppose, on the contrary, that we have a family $\mathcal{F}^{*}=\left\{\Gamma_{1}, \ldots, \Gamma_{m}\right\}, m \geq n$, constructed inductively by (50) and (51) such that it does not cover all vertices. However, by the inductive construction of $\mathcal{F}^{*}$ every vertex is already in some copy of $H$ included in the family $\mathcal{F}^{*}$. A contradiction.

To proceed with the proof we need to establish some notational conventions. Let $u$ be the vertex of $H$ such that $\chi(H \backslash\{u\})=\chi(H)-1$. Set $H^{\prime}=H \backslash\{u\}, h=|V(H)|$, and $e_{H}=|E(H)|$. For $\mathcal{F}$ and a vertex $v$ as in (50), let $N_{v}$ be the set of neighbors of $v, d_{v}=\left|N_{v}\right|$ and $F=\cup_{i=1}^{m} E\left(\Gamma_{i}\right)$. Our analysis will focus on the consideration of the subgraphs $L=G\left[N_{v}\right]$ and $L^{\prime}=\left(N_{v}, E(L) \backslash F\right)$. We extend $\mathcal{F}$ to $\mathcal{F}^{\prime}$ by simply finding a copy of $H$ which contains $v$ but no edges in $F$. Clearly, if there exists a copy of $H^{\prime}$ in $L^{\prime}$, then this $H^{\prime}$ together with $v$ gives a copy of $H$ that extends $\mathcal{F}$. (Note $H^{\prime}$ is a subgraph of $L=G\left[N_{v}\right]$ ).

We have for $|E(L)| \geq \frac{d_{v}}{2}\left(\delta-\left(n-d_{v}\right)\right)$. Since $\delta \geq\left(\frac{\chi-2}{\chi^{-1}}+\epsilon\right) n$ is equivalent to $\delta-n \geq-\frac{1}{\chi-2} \delta+\epsilon n \frac{\chi-1}{\chi-2}$, we get

$$
\begin{aligned}
|E(L)| & \geq \frac{d_{v}}{2}\left(\delta-\left(n-d_{v}\right)\right) \\
& \geq \frac{d_{v}}{2}\left(d_{v}-\frac{1}{\chi-2} \delta+\epsilon n \frac{\chi-1}{\chi-2}\right) \\
& \geq \frac{d_{v}^{2}}{2} \cdot \frac{\chi-3}{\chi-2}+\epsilon n \frac{d_{v}}{2} \cdot \frac{\chi-1}{\chi-2} .
\end{aligned}
$$


Since we are assuming that $|\mathcal{F}|<n$, we have

$$
|F \cap E(L)| \leq|F| \leq e_{H} n
$$

and it follows

$$
\begin{aligned}
\left|E\left(L^{\prime}\right)\right|= & |E(L)|-|F \cap E(L)| \\
\geq & \frac{d_{v}^{2}}{2} \cdot \frac{\chi-3}{\chi-2}+\epsilon n \cdot \frac{d_{v}}{2} \cdot \frac{\chi-1}{\chi-2}-e_{H} n \\
\geq & \left(\begin{array}{c}
d_{v} \\
2
\end{array}\right) \cdot \frac{\chi-3}{\chi-2}+\frac{1}{2} \epsilon\left(\begin{array}{c}
d_{v} \\
2
\end{array}\right) \frac{\chi-1}{\chi-2} \\
& +\left(\frac{1}{2} \epsilon\left(\begin{array}{c}
d_{v} \\
2
\end{array}\right) \frac{\chi-1}{\chi-2}+\frac{d_{v}}{2} \cdot \frac{\chi-3}{\chi-2}+\epsilon \frac{d_{v}}{2} \cdot \frac{\chi-1}{\chi-2}-e_{H} n\right) .
\end{aligned}
$$

Letting $\epsilon^{\prime}=\frac{1}{2} \cdot \frac{\chi-1}{\chi-2} \cdot \epsilon$ and $d_{v}$ be large enough (i.e. $n$ large enough), we conclude that

$$
\frac{1}{2} \epsilon\left(\begin{array}{c}
d_{v} \\
2
\end{array}\right) \frac{\chi-1}{\chi-2}+\frac{d_{v}}{2} \cdot \frac{\chi-3}{\chi-2}+\epsilon \frac{d_{v}}{2} \cdot \frac{\chi-1}{\chi-2}-e_{H} n \geq 0
$$

and thus, $\left|E\left(L^{\prime}\right)\right| \geq\left(\frac{\chi-3}{\chi-2}+\epsilon^{\prime}\right)\left(\begin{array}{c}d_{v} \\ 2\end{array}\right)$. By the Erdös - Stone theorem there exists a copy of $H^{\prime}$ in $L^{\prime}$. Taking this copy of $H^{\prime}$ together with $v$ and edges needed gives us a new copy of $H$ by which we extend $\mathcal{F}$ to $\mathcal{F}^{\prime}$.

Proof of Theorem 5. We are going to determine the exact value of $f(n, 3, k), k \geq$ $\frac{n-1}{2}$ and $n \geq 6$. First, note that in any $\left(K_{3}, \infty\right)$-vertex-cover of a graph $G$ on $n$ vertices no vertex lies in more than $\frac{n-1}{2}$ copies of $K_{3}$. In order to get a tight result we assume $G$ is a graph on $n$ vertices with $\delta(G) \geq\lceil n / 2\rceil+1$. Let $\mathcal{F}=\left\{\Gamma_{1}, \ldots, \Gamma_{m}\right\}$ and $v$ be as in (50) with $H=K_{3}$. We use the notation introduced in the proof of Theorem 6. Unlike in the proof of Theorem 6, in order to get a tight result it does not suffice to simply add a new $K_{3}$ to $\mathcal{F}$. Our argument includes consideration of several different kinds of modifications of $\mathcal{F}$.

It follows from our minimal degree condition that

$$
d_{L}(x) \geq 2, \quad \text { for all } x \in N_{v}
$$

If there is an edge in $L$ not contained in $F=\cup_{i=1}^{m} E\left(\Gamma_{i}\right)$ then this edge together with $v$ gives an extension of $\mathcal{F}$ that contains $v$, and therefore we can assume

$$
E(L) \subset F \text {. }
$$

It follows from (52) and (53) that $|F \cap E(L)| \geq d_{v}=\left|N_{v}\right|$, and therefore

$$
3\left|\mathcal{F}_{3}\right|+\left|\mathcal{F}_{2}\right| \geq d_{v} \geq \frac{n}{2}+1
$$

where $\mathcal{F}_{j}=\{\Gamma \in \mathcal{F}:|V(\Gamma) \cap V(L)|=j\}, j=2$, 3. Since $H=K_{3}$, to simplify the description we identify $\Gamma \in \mathcal{F}$ with its vertex set, i.e. $\Gamma=\left\{x_{1}, x_{2}, x_{3}\right\}$. Consider 
$\Gamma_{A}=\left\{x_{1}, x_{2}, y\right\} \in \mathcal{F}_{2}$ with $x_{1}, x_{2} \in N_{v}$ and $y \in V(G) \backslash\left(N_{v} \cup\{v\}\right)$. If there exists $\Gamma_{B} \in \mathcal{F}, \Gamma_{B} \neq \Gamma_{A}$, such that $y \in \Gamma_{B}$ then $\left(\mathcal{F} \backslash\left\{\Gamma_{A}\right\}\right) \cup\left\{\left\{x_{1}, x_{2}, v\right\}\right\}$ is an extension of $\mathcal{F}$ containing $v$. Therefore, we can assume

$$
\left|\mathcal{F}_{2}\right| \leq\left|V(G) \backslash\left(N_{v} \cup\{v\}\right)\right| \leq \frac{n}{2}-2,
$$

because otherwise there exists a pair $\Gamma_{A}, \Gamma_{B} \in \mathcal{F}, \Gamma_{A}=\left\{x_{1}, x_{2}, y\right\}, \Gamma_{B}=\left\{z_{1}, z_{2}, y\right\}$ as above. It follows from (54) and (55) that $\left|\mathcal{F}_{3}\right| \geq 1$. Now, consider $\Gamma_{A} \in \mathcal{F}_{3}$. If there exists $\Gamma_{B} \in \mathcal{F}$ such that $\Gamma_{A} \cap \Gamma_{B}=\{x\}$ then $\left(\mathcal{F} \cup\left\{\Gamma_{A} \backslash\{x\} \cup\{v\}\right\}\right) \backslash\left\{\Gamma_{A}\right\}$ is an extension of $\mathcal{F}$ containing $v$. So, we can henceforth assume

$$
\Gamma_{A} \in \mathcal{F}_{3}, \Gamma_{B} \in \mathcal{F} \Longrightarrow \Gamma_{A} \cap \Gamma_{B}=\emptyset .
$$

Once again, we consider $\Gamma_{A}=\left\{x_{1}, x_{2}, x_{3}\right\} \in \mathcal{F}_{3}$. Since $d_{G}\left(x_{i}\right) \geq n / 2+1>3$ (here we use our assumption on $n$ ) there exists $u \in V \backslash\left\{v, x_{1}, x_{2}, x_{3}\right\}$ and $a \neq b \in\{1,2,3\}$ such that $u$ is adjacent to both $x_{a}$ and $x_{b}$. Let $c=\{1,2,3\} \backslash\{a, b\}$ and set

$$
\mathcal{F}^{\prime}=\mathcal{F} \backslash\left\{\Gamma_{A}\right\} \cup\left\{\left\{x_{a}, x_{b}, u\right\},\left\{x_{a}, x_{c}, v\right\}\right\} .
$$

By (56) the family $\mathcal{F}^{\prime}$ is edge-disjoint and covers $v$.

In order to prove the lower bound on $f(n, 3, k)$ we consider the following two graphs. If $n=2 m, H_{n}^{e}$ is the complete bipartite graph on the vertex set $Z_{1} \cup$ $Z_{2},\left|Z_{1}\right|=\left|Z_{2}\right|=m$. In the case $n=2 m+1, H_{n}^{o}$ consists of the edges of the complete bipartite graph on the vertex set $Z_{1} \cup Z_{2},\left|Z_{1}\right|=m+1,\left|Z_{2}\right|=m$. Moreover, if $\left|Z_{1}\right|$ is even, $H_{n}^{o}$ contains edges of a perfect matching of $Z_{1}$ and in the case $\left|Z_{1}\right|$ is odd, $H_{n}^{o}$ contains edges of a maximal matching, say $M$, of $Z_{1}$ together with a single edge $\{x, y\}$ where $x$ is the vertex of $Z_{1}$ which does not belong to $M$ and $y$ is any vertex of $Z_{1} \backslash\{x\}$. Clearly, $\delta\left(H_{n}^{e}\right)=\lceil n / 2\rceil$ and $\delta\left(H_{n}^{o}\right)=\lceil n / 2\rceil$. Further, neither of $H_{n}^{e}$ and $H_{n}^{o}$ contains a $\left(K_{3}, \infty\right)$-vertex-cover because $H_{n}^{e}$ does not contain any copy of $K_{3}$ and $H_{n}^{o}$ contains only at most $\lceil(n+1) / 4\rceil$ copies of $K_{3}$.

\section{References}

[1] N. Alon, J. H. Spencer, The Probabilistic Method, John Wiley and Sons, 1991.

[2] N. Alon, R. Yuster, Almost H-factors in dense graphs, Graphs \& Comb. 8 (1992), 95-102.

[3] N. Alon, R. Yuster, Threshold functions for $H$-factors, Combinatorics, Probability, \& Computing 2 (1993), 137-144.

[4] N. Alon, R. Yuster, H-factors in dense graphs, J. Comb. Theory B, to appear.

[5] B. Bollobás, Random graphs, Academic press, 1985. 
[6] F. Chung, R. Graham, Erdős on graphs - his legacy of unsolved problems, A.K. Peters, 1998.

[7] P. Erdős and A. Rényi, On the existence of a factor of degree one of a connected random graph, Acta. Math. Acad. Sci. Hungar. 17 (1966) 359-368.

[8] A.M. Frieze, On small subgraphs of random graphs, Proceedings of Random Graphs '89, (A.M.Frieze and T.Łuczak Eds.) John Wiley and Sons, 1992, 67-90.

[9] A. Hajnal, E. Szemerédi, Proof of a conjecture of Erdös, Comb. Theory and Appl. II, Colloq. Math. Soc. J. Bolyai 4, North-Holland, 1970, 601-623.

[10] S. Janson, Poisson approximation for large deviations, Random Structures and Algorithms 1 (1990), 221-229.

[11] M. Krivelevich, Triangle factors in random graphs, Combinatorics, Probability, and Computing 6 (1997), 337-347.

[12] D.E. Knuth, R. Motwani and B.G. Pittel, Stable husbands, Random Structures and Algorithms 1 (1990), 1-14.

[13] J. Komlós, G. N. Sárközy, E. Szemerédi, Proof of the Alon - Yuster conjecture, in preparation.

[14] T. Łuczak, On the equivalence of two basic models of random graphs, Proceedings of Random Graphs '87 (M.Karoński, J.Jaworski and A.Ruciński, Eds.) John Wiley and Sons, 1990, 151-157.

[15] A. Ruciński, Matching and covering the vertices of a random graph by copies of a given graph, Discrete Math. 105 (1992), 185-197.

[16] N. C. Wormald, The perturbation method and triangle-free random graphs, Random Structures and Algorithms 9 (1996), 253-270. 
This research was sponsored in part by National Science Foundation (NSF) grant no. CCR-0122581. 